\title{
Tabaco, economía campesina y Capitalismo en los Montes de María, 1850-1930
}

\author{
Wilson Blanco Romero ${ }^{(*)}$
}

Presentado: julio de 2011

Aprobado: septiembre de 2011

\section{Resumen}

La historia de la expansión tabacalera exportadora en la subregión de los Montes de Maria, conocida también con los nombres de Serranía de San Jacinto y de Sierra de María ${ }^{1}$, con centro en la antigua Provincia del Carmen de Bolívar en el Caribe colombiano, tiene como rasgo peculiar el hecho de la asociación entre capital y economía campesina. A diferencia de los centros tabacaleros andinos, como Ambalema Tolima, en donde el capital se asocia con la propiedad terrateniente en el marco de la hacienda. Nuestra investigación estudia las circunstancias históricas que explican este hecho de una economía comarcal que produce tabaco negro en rama para la exportación, en el contexto general de una región como la costa norte de Colombia donde el latifundio y la hacienda han tenido tanto peso en la economía agraria y la problemática social.

Palabras claves:

Montes de María, economía tabacalera, pequeña economía campesina, cosecheros.

\section{Abstract}

The history of tobacco export expansion in the sub-region of Montes de Maria, also known by the names of Serrania de San Jacinto and Sierra Mary, with its center in the former province of Carmen de Bolivar in the Colombian Caribbean, has the feature the peculiar fact of the association between capital and peasant economy. Unlike tobacco Andean centers as Ambalema (Tolima), where the capital is associated with land ownership in the ranch. Our research examines the historical circumstances that explain this fact in a regional economy that produces snuff black leaf for export in the overall context of a region like the north coast of Colombia where the large estates and finances have been so much weight on the economy agricultural and social problems.

\section{Key words:}

Montes de María, tobacco economy, small economy peasant harvesters.

${ }^{(*)}$ Magíster en Historia convenio Universidad del Atlántico-Universidad Nacional de Colombia. Profesor titular del Programa de Historia de la Universidad de Cartagena. Miembro del grupo de investigaciones Frontera, sociedad y cultura del Programa de Historia de la Universidad de Cartagena, categoría B, clasificación Colciencias. Ha publicado en Huellas (Universidad del Norte) y El Taller de la Historia (Universidad de Cartagena).

${ }^{1}$ B. Le Roy Gordon, El Sinú. Geografía humana y ecológica, Bogotá, Carlos Valencia eds., 1983, p.14. 
$\mathrm{E}$ problema que nos planteamos en este artículo es el de la identificación de los rasgos económico-sociales que caracterizan el desarrollo de la industria tabacaleraexportadora en la región de los Montes de María, con centro en la otrora Provincia del Carmen de Bolívar. Al examinar este problema y su evolución en el periodo indicado, hemos encontrado, precisamente, una persistente y estructural asociación entre economía campesina y capitalismo. Hecho que peculiariza el caso de la economía del tabaco de la región del Carmen ante el de otras regiones, como la de Ambalema (Tolima), durante el siglo XIX. En donde, si bien se hace presente el capitalismo, la base agrícola de la producción tabacalera estaba fundada en la propiedad y control terrateniente ${ }^{2}$, no en la producción campesina.

\section{Contexto histórico y espacial}

La subregión de los Montes de María, conocida también en la geografía de Colombia cono serranía de San Jacinto, adquiere importancia en la vida económica de la costa Caribe desde tiempos coloniales, mucho antes de que su tabaco le diera fama en los mercados europeos. Ello se debe, no sólo a la abundancia de tierras fértiles para la agricultura y la ganadería, sino sobre todo a su estratégica y crucial posición a medio camino entre el Valle del Sinú y las sabanas de Tolú o de Corozal y el puerto de Cartagena. Dichas sabanas, principalmente las del Sinú, constituyeron la despensa donde se abastecía la Plaza fuerte de Cartagena desde tempranos tiempos coloniales, por vía marítima. Circunstancias determinadas forzaron a que en la segunda mitad del siglo XVIII, en el marco de la política borbónica de fomento económico del imperio y ante los asedios de piratas, corsarios y grandes escuadras navales de la potencias enemigas que aislaban dicha plaza fuerte por vía marítima, se abriera un camino o ruta terrestre entre Cartagena y su hinterland sabanero, a efectos de asegurar el avituallamiento de la Plaza Fuerte en tiempos de guerra ${ }^{3}$.

Es en esas circunstancias en que se produce la epopeya pobladora de Don Antonio de la Torre y Miranda, que en la misión de abrir dicho camino funda alrededor de 40 pueblos entre Cartagena y las sabanas del Sinú, en una cadena que va de los aún hoy corregimientos de Ternera y Pasacaballos al norte en las goteras de Cartagena hasta Momil en el sur del departamento de Córdoba, atravesando longitudinalmente los Montes de María en cuyas serranías se ubican 5 de esos pueblos, como son de norte a sur: San Cayetano, San Juan Nepomuceno, San Jacinto, Nuestra Señora del Carmen hoy El Carmen de Bolívar, y San Francisco de Asís, hoy Ovejas ${ }^{4}$.

El asunto es que, dicha subregión, dada su ubicación y la feracidad de sus tierras desde tempranos tiempos, y atravesada por tan importante camino (convertido hoy en la carretera

\footnotetext{
${ }^{2}$ René de la Pedraja, "Los cosecheros de Ambalema: un esbozo preliminar" en Anuario Colombiano de Historia Social y de la Cultura No 9, Bogotá, Universidad Nacional, 1979.

${ }^{3}$ Ver Antonio de la Torre y Miranda, "Noticia individual de las poblaciones nuevamente fundadas en la Provincia de Cartagena" en Proa, Bogotá, enero de 1972.

${ }^{4}$ Alfonso Múnera, "Ilegalidad y frontera 1770-1800" en Adolfo Meisel (ed.), Historia económica y social del Caribe colombiano, Barranquilla, Uninorte, 1994, p.118.
} 
Troncal del Caribe) se convierte en una importante zona de encrucijada en el entramado de las rutas terrestres intercomarcanas entre Cartagena y su hinterland agroganadero, desarrollando una temprana vocación agro-mercantil. Al punto de que una parte del maíz que a fines del siglo XVIII se consumía en la Provincia de Cartagena provenía, precisamente, de los Montes de Maria ${ }^{5}$.

\section{El tabaco}

Ahora, en lo que a tabaco se refiere, encontramos que, en el desarrollo de la historia económica de Colombia en lo que hace a los productos de la agricultura tropical orientados a la exportación, al tabaco le cabe un papel pionero mucho antes que el café. Como que constituyó, hacia mediados del siglo $\mathrm{XIX}$, la primera gran expansión productora $\mathrm{y}$ exportadora que vinculó la economía colombiana al moderno mercado mundial suministrando la carga de bajada que en términos de sostenibilidad permitió la regularización de la navegación a vapor por el rió Magdalena ${ }^{6}$, en la comunicación entre el país andino y su fachada portuaria del Caribe. Con lo cual, se dan las condiciones económicas y de modernización infraestructural básicas para lo que sería el llamado modelo agro exportador de desarrollo. Recordemos además, que durante la época colonial, la fumable solanácea, desde la segunda mitad del siglo XVlll llegó a tener una importancia fiscal de primer orden bajo el régimen de monopolio oficial conocido como el estanco del tabaco ${ }^{7}$.

En el marco de la gran expansión decimonónica, que tiene lugar en la segunda mitad de dicha centuria, la región del Carmen o de los Montes de Maria, dada su ubicación geográfica en la Costa Caribe, relativamente bastante próxima a la arteria del río Magdalena que posibilitaba su rápida conexión con los puertos de salida, va a disponer de importantes ventajas comparativas ante las otras regiones tabacaleras en el interior andino ${ }^{8}$. De tal manera que, entrada la sexta década de dicho siglo, se consolida y logra la primacía sobre el que había sido el principal centro tabacalero desde los tiempos coloniales, como lo era Ambalema en el alto Magdalena. Para el cierre del siglo, tras crisis insuperables ante la baja de precios y la aparición de nuevos competidores en el mercado europeo, tanto Ambalema como los demás centros productores para la exportación, del interior andino, se

\footnotetext{
${ }^{5}$ A. Múnera, "Ilegalidad y frontera 1770-1800”, p.129.

${ }^{6}$ Luís Eduardo Nieto Arteta, Economía y Cultura en la Historia de Colombia, Bogotá, El Ancora Eds., 1983, pp. 197-198; Luís F. Sierra, El tabaco en la economía colombiana del siglo XIX, Bogotá, Universidad Nacional de Colombia, 1971, p.97; y Fabio Zambrano, "La navegación a vapor por el rió Magdalena" en Anuario Colombiano de Historia Social y de la Cultura № 9, Bogotá, Universidad Nacional de Colombia, 1979, p. 64

${ }^{7}$ Luis Ospina Vásquez, Industria y protección en Colombia. 1810-1930, Medellín, Faes, 1979, p. 63.

${ }^{8}$ John Parker Harrison, The Colombian Tobacco Industry: from government monopoly to free trade, 17781876, tesis doctoral inédita, Berkeley, Universidad de California, 1951, copia dactilográfica, en inglés, del Cede de la Universidad de los Andes, Bogotá, 1969, pp. 246-248
} 
ven aniquilados y desaparecen como tales ${ }^{9}$; quedando en pie desde las primeras décadas del siglo XX sólo la región tabacalera exportadora del Carmen ${ }^{10}$.

Un factor que explica la continuidad del tabaco de El Carmen tras el cambio de siglo, por encima de guerras y crisis internas y externas, es, como lo indica José Antonio Ocampo, su capacidad para adaptarse a las épocas de precios bajos en un mercado internacional cada vez mas competido ${ }^{11}$. Sumado a ello la abundancia de suelos en la comarca de los Montes de María aptos para producir un excelente tabaco negro en rama tipo exportación ${ }^{12}$, con una calidad mejorada, al parecer desde los mismos tiempos coloniales, con semilla traída de $\mathrm{Cuba}^{13}$.

Finalmente constatemos aquí, para efecto de lo que sigue, que a lo largo del periodo del que nos ocupamos, la economía tabacalera de la región del Carmen estuvo al servicio de la acumulación de renta capitalista. Atrayendo desde muy temprano la inversión de capital mayoritariamente extranjero, que operando a través de empresas conocidas como "Casas compradoras" o mas apropiadamente, hoy día, como Tabacaleras acopiaban el tabaco producido en la subregión y, tras procesarlo, lo destinaban especialmente a la exportación en rama de miles de toneladas a Europa ${ }^{14}$. Es, la inmigración comercial o empresarial que da lugar a la presencia extranjera en la comarca de empresarios como los Hollman, los Held, los Hamburguer y otros alemanes; los Frieri, Gallo, Volpes, y otros italianos; o los Barnier franceses, o los siriolibaneses, y algunos holandeses. Tema del que nos hemos ocupado con detenimiento en otro texto, ya publicado ${ }^{15}$.

\footnotetext{
${ }^{9}$ L. Sierra, El tabaco en la economía colombiana del siglo XlX; Eduardo Posada, El Caribe colombiano. Una historia regional, (1870-1950), Bogotá, El Ancora-Banco de la República, 1998; J. Harrison The Colombian Tobacco Industry; y José Antonio Ocampo, Colombia y la economía mundial 1830-1910, Universidad Nacional- Siglo XXI eds., Bogotá, 1984.

${ }^{10}$ Wilson Blanco, "La exportación tabacalera de El Carmen de Bolívar en los albores del siglo XX: guerra y tabaco", en El Taller de la Historia N N $^{\circ}$, Cartagena, Universidad de Cartagena, 2001.

${ }^{11}$ Ver J. A. Ocampo, Colombia y la economía mundial, p. 241, quien al refirerirse a la fuerte reacción del tabaco Carmen a principios de los años 70 del siglo XIX, tras la caída definitiva de Ambalema, escribió: "El Carmen había demostrado por primera vez su capacidad para adaptarse a los bajos precios internacionales".

12 J. Harrison, The Colombian Tobacco Industry, pp. 40-41

${ }^{13}$ Wilson Blanco, "El tabaco de El Carmen en la Costa del Caribe colombiano. La vieja data de su estirpe cubana”. Ponencia leída en el VII Seminario Internacional de Estudios del Caribe en Cartagena, agosto de 2005. Inédito.

${ }^{14}$ W. Blanco, "La exportación tabacalera de El Carmen de Bolívar en los albores del siglo XX: Guerra y tabaco".

${ }^{15}$ Wilson Blanco, "Comercio e inmigración en la provincia costeña. Los italianos de El Carmen de Bolívar. El caso de los Volpe" en El Taller de la Historia N $^{\circ}$ 2, Cartagena, Universidad de Cartagena, 2002.
} 


\section{Economía campesina y capitalismo}

La revisión, tanto de las fuentes documentales, como los libros de protocolo de la Notaría Única de la antigua Provincia de El Carmen fechados desde la mitad del siglo XIX, como las fuentes orales, dejan ver de manera fehaciente que en la economía tabacalera de la región de El Carmen la presencia del capital no estaba ligada a la propiedad terrateniente sino a la economía campesina.

Ahora preguntémonos ¿Cómo es que la economía campesina aparece como forma predominante en la base económico-social de la producción agrícola del tabaco? La cuestión es, que el dominio de la economía campesina en la subregión es muy anterior a la conformación de la economía tabacalera. Desde los mismos tiempos del fundador de pueblos Antonio de la Torre y Miranda, durante la colonia, se dan las condiciones para que esto sea así. Dado que, en lo que hace al caso particular de lo que nosotros llamamos comarca montemariana o Montes de María, a diferencia de lo que sucede en las tierras bajas del Sinú, el Magdalena y el Cauca, no tiene lugar el desarrollo de una economía agraria latifundista basada en la expansión de la hacienda esclavista ${ }^{16}$ sino que, lo que se da es una colonización de blancos pobres basada en la pequeña producción campesina, que persiste mas allá del siglo XVIII ya en los tiempos republicanos e incluso veinte seculares, como lo hemos podido constatar en nuestra investigación.

Al intentar el análisis histórico-económico de la estructura que sustenta la producción de tabaco negro en rama en la región del Carmen, hay que partir del hecho de que nos encontramos ante una economía regional, de base campesina, pero que produce para el mercado. Tanto para el mercado nacional como para el mercado exterior; pero, especialmente para este último. Empezamos constatando así, entonces, el carácter mercantil de dicha economía tabacalera: la producción de tabaco por los campesinos o cosecheros pero no para el autoconsumo sino para el mercado, especialmente el externo. Todo esto, sometido a la supremacía del régimen capitalista de producción tanto a nivel nacional como mundial. Pero desde lo que se conoce como la periferia, en donde dicho régimen capitalista de producción no alcanza su desarrollo pleno e integral, dando lugar a la sobré vivencia de formas sociales de producción de carácter precapitalista como la pequeña producción campesina, pero que funcionan al servicio de la acumulación de renta capitalista.

El establecimiento y consolidación de tal economía, requiere no sólo de capital de inversión, sino de que éste encuentre las condiciones suficientes para su acumulación con una tasa de ganancia o rentabilidad que garantice su permanencia de manera ventajosa.

${ }^{16}$ Adolfo Meisel, "Esclavitud, mestizaje y haciendas en la provincia de Cartagena 1533-1851" en Gustavo Bell (ed.), El Caribe colombiano, Barranquilla, Universidad del Norte, 1988; Hermes Tovar, Grandes empresas agrícolas y ganaderas. Su desarrollo en el siglo XVIII, Bogotá, Ciec, 1980; Orlando Fals Borda, Capitalismo, hacienda y poblamiento en la Costa Atlántica, Bogotá, Ed. Punta de Lanza, 1976. 
¿Cómo se ha logrado esto? A través del desarrollo histórico y la práctica consuetudinaria de determinadas formas sociales de producción que han surgido en el contexto comarcal.

Para avanzar hacia el examen histórico de ese proceso partimos de que cualesquiera que sean las formas sociales de la producción, sus factores son siempre dos: los medios de producción y los trabajadores o productores directos. Veámoslo, entonces, para nuestro caso como se ha dado históricamente la vinculación de los productores directos como fuerza de trabajo o mano de obra, a la tierra, para hacer uso de ella como medio de producción básico para la producción de tabaco negro en la región del Carmen.

Al respecto hay que tener en cuenta, que en la comarca montemariana desde la misma época de la fundación de pueblos, hacia fines del siglo XVIII, sus habitantes (mestizos y blancos pobres en su gran mayoría) tuvieron facilidad para acceder gratuita y libremente a la tenencia y uso de la tierra, tratándose de una zona de colonización tardía con abundancia de baldíos. Recordemos que, la campaña pobladora de la Torre y Miranda, en la antepenúltima década del siglo XVIII, se hizo con base en la asignación de solares a los vecinos de cada fundación para fijar vivienda, y parcelas de tierra para cultivo y cría de ganado. Lo que desde entonces dio lugar a la formación de una agricultura de pequeña producción campesina entre iguales.

Ahora, si bien es cierto que en la comarca hubo un temprano desarrollo agroganadero, este no alcanzó una dimensión productiva y mercantil tal, como para impedir que durante todo el siglo XIX y hasta entrado el siglo XX se mantuvieran grandes extensiones de tierras no ocupadas y baldías; que de hecho, resultaran de fácil, libre y pacífica posesión.

Lo cierto es que, al parecer, en la comarca de los Montes de María a diferencia de otras subregiones de la costa Caribe, como las hoy sabanas de Sucre y Córdoba, la depresión Momposina y los valles del Sinú y el San Jorge ${ }^{17}$, no se dio un proceso de gran expansión hacendaría o terrateniente que, sobre la base de una gran concentración de la propiedad territorial, conllevara una temprana y radical expropiación, que limitara de manera significativa el fácil acceso y uso campesino del suelo. Así, en uno de los pocos estudios conocidos sobre el tema de la ocupación de baldíos en el Caribe colombiano se puede advertir como, nuestra subregión no aparece entre aquellas en las cuales se dio la adjudicación a particulares de grandes extensiones de baldíos como factor de concentración y exclusión de la posesión y uso del suelo durante el siglo XIX, como si fue el caso de otras partes de la Costa, especialmente la zona correspondiente a la jurisdicción de río Sinú como lo muestra dicho estudio ${ }^{18}$.

Lo anterior no niega, por supuesto, que en la subregión no se hubiera dado cierto proceso de expansión y concentración de la propiedad hacendaría y terrateniente, dando lugar a la

${ }^{17}$ Para dichas regiones se pueden ver los estudios citados supra de Fals Borda, Hermes Tovar y Adolfo Meisel.

${ }^{18}$ Hermes Tovar, "Los baldíos y el problema agrario en la costa Caribe de Colombia" en Fronteras $\mathrm{N}^{\mathrm{o}} 1$, Bogotá, Centro de Investigaciones de Historia Colonial, 1997, pp. 38-44. 
formación de haciendas o latifundios, inexplotados en su gran parte. Pero ese proceso, por lo que hemos visto, fue bastante tardío acá. Haciéndose evidente en la revisión de la documentación notarial, ya bien entrado el siglo XX; cuando en la comarca, el auge tabacalero, de origen decimonónico, se acompaña de una considerable expansión de la ganadería en las condiciones de un mayor desarrollo demográfico y mercantil para la época.

Lo cierto es que a mediados del siglo XIX, cuando surge el auge exportador tabacalero su desarrollo productivo va a tener lugar es bajo el predominio de esa estructura agraria de pequeña producción campesina. En donde encaja perfectamente la práctica tradicional del cultivo hortense y artesanal del tabaco negro de la región del Carmen. Dicho auge llevó, a que mucha gente aprovechando el fácil acceso a la libre posesión y uso del suelo se dedicara a cultivar y producir para el mercado un tabaco que, como el tabaco negro tipo cuba o cubita, dada su productividad y gran valor en las condiciones del medio natural Monte mariano, no necesitó de grandes o ni siquiera medianas unidades de explotación para que se convirtiera en el privilegiado y fundamental sustento económico del pueblo cosechero, requiriendo poca inversión de capital-dinero. De allí que se convirtió en un cultivo masivo de pobres, por excelencia.

Las mismas características agrológicas del cultivo del tabaco Carmen, de hecho se convirtieron en un factor de desestímulo a la expansión de la propiedad terrateniente, como forma de producción tabacalera. Así, no obstante la intensa, cuantiosa y rápida expansión que llevó a que la región en menos de 3 lustros, tras la abolición del monopolio, se convirtiera en primera productora y exportadora de tabaco a partir del año 1863, desplazando a la región de Ambalema Tolima, no se requirió de la apropiación y cultivo de latifundios o haciendas de miles de hectáreas para alcanzar los mayores volúmenes de producción con base en una estructura agraria tipo terrateniente. Aunque carecemos de un estudio específico por falta de información detallada, apoyándonos en lo que hemos podido captar de la revisión de la documentación notarial y de algunos informes en la prensa oficial, presumimos que el auge tabacalero decimonónico, y aún su continuación entrado el siglo XX, fue con base en la pequeña producción campesina de tipo parcelario; blindada, digámoslo así, por la abundancia de tierras aptas y disponibles de fácil y libre ocupación; y en condiciones, de una relativamente débil presión demográfica que además de otros factores de orden económico, que habría que precisar, contribuyeron a una limitada o lenta dinámica en la expansión de la frontera agraria.

Así, tenemos tempranas pruebas documentales que muestran que los terrenos aledaños al Distrito del Carmen, que catapultado por el desarrollo mercantil del auge tabacalero en sólo una década pasó de parroquia a villa y de villa a capital de la provincia de su nombre, hacia fines de la década de los 50s. se hallaban ocupados por parcelas de tabaco, explotadas por campesinos pobres; que desde entonces, ya comprometían su producción por adelantado para entregarla a capitalistas acaparadores directamente o por intermedio de los famosos corredores, a cambio de lo que se llamo "avances", en dinero o especies. 
En el temprano año de 1859, el señor Ignacio Suárez, de Zambrano, como garantía de pago para respaldar la deuda de "\$80.00 moneda de lei” que debía pagar al Sr. José Mercedes Álvarez, en el término de 10 meses, con 10 quintales de tabaco, le tocó hipotecar los bienes siguientes de su propiedad:

dos burras mochas una color moro blanco i el otro moro prieto...la mitad de la casa morada de madera i palma sará... i un tabacal situado en la fracción de Cansaculo... de mas de media cabuya de tabaco sembrado, fuera de otra parte mas de terreno desmontado que también deberá sembrar de tabaco...linda por el Este con el arroyo de Alférez[ que pasa aún lado de la actual población de El Carmen ], por el Oeste con el tabacal de la Sra. Seledonia Serrano, por el Norte con el camino de la fracción expresada i por el Sur punta de monte por el medio con el tabacal de Esteban García... ${ }^{19}$

Mientras que en declaración testamentar el Sr. Santiago Torres, vecino del Distrito del Carmen manifiesta, que "posee una cabulla de tabacal situada en el paraje de Bonito abajo terrenos de este Distrito, linda por el Oriente con una punta de tierra firme...y por el Sur con el tabacal del Sr. Prudencio Torres". Allí vemos, pues, tabacales por aquí y tabacales por allá; y además, puntas de monte desmontables por un lado y por el otro.

Pero el siguiente pasaje, del informe de un gobernador decimonónico de la Provincia del Carmen, a principio de la década de los sesenta del mismo siglo, es aún mas elocuente en relación a lo que hemos querido mostrar:, "La preciosa libertad sancionada en nuestra legislación[ se refiere a la abolición del estanco o monopolio del tabaco ], la feracidad de los terrenos de la provincia y su grande extensión inculta por falta de brazos que con sólo la ocupación adquieren el derecho a explotarlos, deben llamar la atención de la Asamblea y del poder ejecutivo a favor de la emigración",20.

Claro está que, andando el tiempo dado el desarrollo mercantil generado por el mismo auge productor y exportador de tabaco con la presencia de grandes negociantes y capitalistas tanto nacionales como extranjeros y con el desarrollo de la expansión ganadera, hacia fines del siglo XIX se fue entrando en un proceso de expansión, especulación y concentración de la propiedad territorial que fue llevando a que además de los campesinos cosecheros, que seguían y aún siguen produciendo el tabaco en terrenos propios, u ocupando libre y gratuitamente las áreas desocupadas, aparecieran otros cosecheros que para cultivar el tabaco les tocaba acceder a la tierra de quienes la tenían ya apropiada, fueran terratenientes(mas o menos latifundistas) o simples campesinos medios.

\footnotetext{
${ }^{19}$ Archivo Histórico de Cartagena (Colombia), Fondo Notarial, Notaria Única del Carmen de Bolívar, No 50 de julio 19 de 1859. (En adelante esta fuente se ciatará por las iniciales AHC, CB).

${ }^{20}$ Gaceta Oficial del Estado Soberano de Bolívar, Cartagena, septiembre 30 de 1862.
} 
La cuestión es que, no obstante los progresos de la expansión ganadera, aún a lo largo de las primeras décadas del siglo XX se mantuvieron condiciones que permitían el fácil y aún libre acceso al uso y posesión del suelo ${ }^{21}$ apto para el cultivo de tabaco, dado que la abundancia de terrenos baldíos y públicos se mantuvo tardíamente, hasta bien entrado el siglo XX, en el ámbito rural no solo de la región de El Carmen sino de lo que fue el antiguo Departamento de Bolívar ${ }^{22}$. Como lo demuestran, avisos de la prensa oficial como los que a continuación copiamos, el primero aparecido en septiembre de 1913:

\title{
AVISO OFICIAL
}

Se hace saber a todas las personas que tienen en la Gobernación solicitudes de adjudicación de terrenos baldíos, que deben apoderar a alguna persona en esta ciudad que la represente para los efectos de notificaciones, suministro de papel sellado, estampillas, pagos de derechos, y al no hacerlo sufrirán los perjuicios consiguientes... ${ }^{23}$

El otro apareció en octubre de 1920, como podemos leer:

\begin{abstract}
SECCION VARIA
Advertencia importante sobre terrenos baldíos a ganaderos y agricultores nacionales y extranjeros.

Para la compra de tierras declaradas baldías por el Gobierno deben informarse si el adjudicatario ha cumplido con cultivar siquiera la tercera parte u ocupado con ganado las 2 terceras partes del terreno en el curso de 10 años contados desde la fecha de adjudicación. De lo contrario queda extinguido vuelve a la Nación de IPSO FACTO (Art.56 del Código Fiscal). ${ }^{24}$
\end{abstract}

La revisión de los protocolos de las primeras décadas del siglo XX de la Notaría de El Carmen ha puesto ante nuestra vista numerosas declaraciones extra-juicio de testigos, empleadas para demostrar la propiedad de muchas personas de diversa condición social, pero mas gente humilde, sobre pequeños y medianos predios poseídos de hecho en

${ }^{21}$ De allí, que las referencias y quejas sobre lo que algunos llamaron sistema de cultivo [de tabaco] errante, que aparecen en el siglo XIX y se repiten en el XX, lo que denotan es la persistencia de las condiciones de fácil y libre acceso, posesión y uso del suelo tabacalero por parte del pueblo cosechero, como podemos ver. Así, en 1879 el Gobernador de la Provincia aboga por la superación de dicho sistema, cuando anota: "Si se alcanza a radicar a los cultivadores, ellos ensancharían sus posesiones, adquirirían mejores hábitos de moralidad y serían verdaderamente ricos"; mientras que una generación después, el fiscal de la provincia habla "del cultivo errante, las quemas, los innecesarios grandes desmontes..." y se queja de que en la localidad del Carmen "... se tala y quema sin piedad.. para abrir rozas...”, además: “... la misma asombrosa fertilidad de nuestro suelo en todo el Dpto. y la facilidad que hay aún para trabajar en terreno nuevo, como que son la causa inmediata de ese abandono..., "Carta de José P. Torres al Director del periódico El Porvenir, Cartagena abril 5 de1906 y junio 29 de 1906 publicadas en Sebastián Mesa Merlano, José Prudencio Torres, Cartagena, Talleres El Mercurio, 1938 pp. 200 y 205-208. Las cursivas son nuestras.

${ }^{22}$ Eduardo Posada, "La economía del Caribe colombiano a comienzos del siglo: 1900-1930" en Estudios Sociales No 2, Medellín Faes, 1988, pp. 75-76.

${ }^{23}$ AHC, Gaceta Departamental de Bolivar, Cartagena, septiembre, 3 de 1913.

${ }^{24}$ AHC, Gaceta Departamental de Bolívar, Cartagena, octubre 20 de 1920. 
condición de baldíos y explotados pacíficamente por muchos años de manera libre. Recojamos dos de los tantos casos que registra la documentación notarial.

Julio Moré F. aparece protocolizando con fecha marzo 26 de 1920 un memorial de 8 fojas, en donde 3 personas ofician como testigos, para probar su larga y pacífica posesión de hecho sobre un terreno en la división entre los cerros de "Floral" y "Floralito" en jurisdicción del Distrito de El Carmen con "casa de habitación café, plátano, cacao, caucho, guamo, aguacate y yerba (sic.)" con una superficie de 30 cabuyas $^{25}$.

Mientras que José del Carmen Marín, de San Jacinto, protocoliza una declaración de testigos que había levantado en el juzgado de San Jacinto en su condición de exponente poseedor, para demostrar "su exclusiva propiedad" sobre un terreno colindante por un lado con "el Retiro denominado Matuya... de 20 cabuyas cercado con alambre de púas, sementera de maíz, y terreno trabajado para sembrar tabaco" ${ }^{\text {26 }}$.

Este último caso, especialmente, nos proporciona una demostración palmaria de cómo ese "pueblo cosechero" de la comarca carmera, al cual se refiere el eminente abogado carmero Manuel Edmundo Mendoza en su artículo de La Patria en $1929^{27}$, venia cultivando tabaco de tiempo atrás en terrenos de acceso libre, ocupados de hecho como baldíos nacionales; y que, con actos notariales como los que acabamos de citar, se buscaba asegurar su propiedad y hacerlos expeditos para la especulación del suelo, en momentos en que la expansión de la frontera agraria ya dinamizaba fuertemente el mercado de tierras en estas regiones del Departamento de Bolívar ${ }^{28}$.

Pero, hacia los años 20s., cuando el avance del proceso de concentración y acaparamiento latifundista de la propiedad privada de la tierra apta para el cultivo del tabaco, dinamizado dicho avance por la expansión ganadera que conllevó la expansión de la frontera agraria ${ }^{29}$, hizo que gran parte del denominado "pueblo cosechero" perdiera la posibilidad de acceder al uso de terrenos propios o a la posesión y uso de baldíos adecuados para dicho cultivo, empieza a tomar auge, en la región de El Carmen, un particular sistema de arrendamiento que podríamos llamar de "tierra por pasto" que tipifica un cierto maridaje entre ganadería y tabaco; a través del cual, el campesino o cosechero en virtud de un contrato por lo general verbal, obtiene el uso de la tierra "arrastrojada"( o sea convertida en rastrojo) del terrateniente-ganadero por un termino comúnmente de dos años; con el compromiso de devolverla hecha potrero( o sea sembrada en pasto para el ganado ).

\footnotetext{
${ }^{25}$ AHC, CB, No 83 marzo 26 de 1920.

${ }^{26}$ AHC, CB, N ${ }^{\circ} 140$, mayo 25 de 1920 . Las cursivas son nuestras.

${ }^{27}$ AHC, “La industria del tabaco en El Carmen", en La Patria, Cartagena, julio 2 de 1929.

${ }^{28}$ E. Posada, El Caribe colombiano, cap. 2 "La ganadería"

${ }^{29}$ Lo que formó parte del proceso de "la expansión de las haciendas ganaderas en las llanuras del Caribe [colombiano]" que de paso menciona Catherine LeGrand, "Los antecedentes agrarios de la violencia: El conflicto social en la frontera colombiana, 1850-1936", en Gonzalo Sánchez y Ricardo Peñaranda (comps.), Pasado y presente de la violencia en Colombia, Bogotá, Cerec, 1991, p. 129.
} 


\section{Conclusión}

Hemos estudiado, como en la subregión de los Montes de María o comarca montemariana en la costa Caribe de Colombia, entre mediados del siglo XIX y los inicios del siglo XX, la presencia del capital vinculado a la industria del tabaco negro en rama para la exportación, aparece asociada es a la pequeña producción campesina, y no a la producción hacendaría o terrateniente como ocurre en los centros tabacaleros del interior andino, como el caso de Ambalema que ha sido estudiado por René de la Pedraja Toman en un artículo del Anuario de Historia Social y de la Cultura de la Universidad Nacional titulado "Los cosecheros de Ambalema: un esbozo preliminar, ${ }^{30}$.

Las circunstancias históricas que explican este hecho están dadas por la ausencia de la expansión terrateniente y hacendaría en la comarca monte mariana, que sólo se va a dar muy tardíamente ya bien entrado el siglo XX. A diferencia de lo que sucede con la temprana expansión de la hacienda ganadera en otras partes de la costa Caribe, como es el caso del valle del San Jorge, el Sinú y la depresión Momposina; como ha sido estudiado por Orlando Fals Borda y Adolfo Meisel en textos muy conocidos. Diferente circunstancia, que permite entender el marcado peso del conflicto por la tierra en estas subregiones costeñas que abarca casi todo el siglo $\mathrm{XX}$, tan débil en la subregión que nosotros estudiamos. De todos modos, esa tardía expansión de la hacienda ganadera genera nuevas formas o relaciones de producción en el marco de la economía del tabaco en los Montes de María, hacia los años 30s del siglo XX, como vimos al final de la ponencia.

${ }^{30}$ Ver nota 1 supra 